\title{
The Centre for Chemical Sensors and Chemical Information Technology (CCS) in Micro- and Nanotechnology
}

\author{
Ursula E. Spichiger-Keller and Prisca Schär-Zammarettia
}

\begin{abstract}
A selection of the most challenging projects in micro- and nanotechnology tackled at CCS during the last few years are discussed. The ultimate challenge was to localize voltage-gated and ligand-gated ion channels on membranes of living cells by ion-selective AFM-tips. In order to address this goal, a procedure to simulate the ion transport over ion-channels was developed. Two types of living cells were investigated; Madin-Darby canine kidney (MDCK-F) cells and adult rat cardiomyocytes (ARC). The results of force microscopy were compared to the patch clamp technique and to immunoselective staining and localization by confocal laser scanning microscopy (CLSM). The lateral resolution of the AFM imaging technique, the rate-limiting steps, the force applied to the cells by the AFM-cantilever and the mechanism of the detection principle are addressed.
\end{abstract}

Keywords: Chemically modified cantilevers · Gas sensor array · Integrated optical sensors . Ion-selective AFM-tip · Ion-selective microelectrodes

\section{Introduction}

CCS is a self-supporting ETH-group which belongs to the Department of Chemistry and Applied Biosciences. The Centre was founded in 1994 by U. Spichiger. The question 'Why is an ETH group like CCS located at Technopark Zurich?' was answered in CHIMIA in 2003 [1]. An overview on projects running at $\mathrm{CCS}$, on its mission and its strategy was presented in CHIMIA in 1997 and 1999 and is accessible on the homepage of CCS at www.chemsens.pharma.ethz.ch) [2][3].

\section{Core competences are:}

- Scientific investigations of novel molecular recognition schemes to be implemented in chemical sensors, biosen-

${ }^{*}$ Correspondence: Prof. U.E. Spichiger-Keller Center for Chemical Sensors and Chemical Information Technology (CCS)

Swiss Federal Institute of Technology (ETH), Zürich CCS, ETH-Technopark

Technoparkstr. 1

$\mathrm{CH}-8005$ Zürich

Tel.: +41014451231

Fax: +41014451233

E-Mail: spichiger@chemsens.pharma.ethz.ch

http://www.chemsens.ethz.ch

anstitute of Biomedical Engineering

ETH and University of Zurich

Moussonstr. 18

CH-8044 Zürich sors and bioassays especially for neutral molecules such as amines, aldehydes and alcohols.

- The development of sensing devices and analytical procedures to provide qualitative and quantitative chemical information about target compounds present in complex specimens. Developments are focused on devices applied to process monitoring, continuous monitoring, and on-site measurements (screening tests and test strips).

In June, CCS celebrated its 10th anniversary with the Decennial conference where a number of excellent and wellknown scientists presented highlights of their work under the headlines 'Sensors in Medical Diagnostics, Life Science and Industry' and 'Trends in Sensor Research'. The group at CCS has a size of 8 to 15 persons depending on the number of academic guests, Ph.D. as well as semester and diploma students. In 1999 and 2002, two spin-off enterprises SENSORIX and C-CIT AG were founded in order for former students to become entrepreneurs. In the following, some key-projects in micro- and nanotechnology are addressed.

\section{From Micro- to Nanotechnology}

Besides the development of new materials that solve relevant analytical problems, strategies are focused on platform technologies which allow the scientific reputation of the Centre to be sustained and improved but also technologies which are believed to have a high market potential in the future. If attractive to industrial partners, mature technologies are resumed and investigated further in view of a new application and new challenges (for an overview on projects see www.chemsens.ethz.ch). Overviews covering developments of new materials for sensor applications have been published in earlier volumes of CHIMIA [2-4].

\section{From Micro- to Nanotechnology}

Based on a background acquired in the group of Prof. W. Simon, Laboratory of Organic Chemistry at ETH Zurich, the investigation of glass microelectrodes, so-called micropipettes, was continued [5-8]. Some attractive applications such as $\mathrm{pH}$-monitoring within noodles for process control of production of instant noodles (project with Nestec SA, Switzerland), monitoring of magnesium concentrations in rat blood samples of small volume (approx. $10^{-4} 1$ ) were demonstrated [9]. These microelectrodes had attractive features in terms of detection limit, response time, miniaturization and, first of all, its selectivity to specific ions. Nevertheless, the lateral resolution of hundred micrometers and more was too low to enable investigations of structures of relevant biological impact such as ion channels. 
In addition to microelectrodes, the collaboration with Robert Bosch $\mathrm{GmbH}$ in Stuttgart, provided access to optical microsensors for gas-selective detection [10]. Optical microsensors allow continuous monitoring of toxic gases in the range of picomolar concentrations [10], and provide microsensor arrays and microsensors to monitor gases in medical applications [11].

\section{In Nanosciences}

The drawbacks of ion-selective microelectrodes and the basic theories, which did not explain its limitations, stimulated the development of sensors which should provide an answer to some of the questions raised. One such question was: At the boundary of an ion-selective membrane cast onto a solid support, is there really a charge separation and, therefore, is a reproducible boundary potential established? In addition, the lateral resolution of ion-selective microelectrodes in reality was not satisfying even if the concentration resolution was high $\left(10^{-9}\right.$ to $\left.10^{-10} \mathrm{M}\right)$ referred to the ion investigated and the tip diameter was 100 to $500 \mathrm{~nm}$ in the most favourable cases [12][13].

Therefore a microelectrode design, which could be promising for tracing the ion-exchange over ion-channels selectively was sought. Chemically modified AFMcantilevers offered themselves for tracing ion-channels selectively and destructionfree on biological tissues [14]. Atomic Force Microscopy (AFM) became known as a very powerful technology to image molecular structures [15-17]. The imaging process resulted from molecular interactions and molecular forces between the scanning cantilever-tip and the substrate. Pharmacologically and biologically attractive applications were published such as 'measuring the binding strength between cell adhesion proteoglycans' [18], and the 'interactions between specific antigens and antibodies' [19], and tracing the 'reversible unfolding of titin immunoglobulin domains'. In addition, applications of force spectroscopy to polymer surfaces were shown [20]. Correlations between adhesion forces and calculations of Van der Waals interactions were found. Recently two reviews were published which summarize the use of cantilevers as chemical sensors and biosensors [21][22]. Based on the results of early publications about tracing molecular interactions, we postulated that two boundaries which are polarized by specific ion exchange must induce adhesion and rupture forces which are accessible to AFM-technology. Therefore, investigations of AFMtips coated with an ion-selective film in electrolyte solutions were initialized within the SNF-programme NFP 36 which was dedicated to the exploration of the industrial potential of nanotechnology.

\section{The Mechanism of an Ion-selec- tive AFM-Tip is Comparable to a Sodium-Potassium Pump}

The principle of the ion-selective AFMtip responding to specific ions released from a substrate was shown in the last overview in CHIMIA [3] and published in [23]. The proof of principle was shown by adsorption of a potassium- and sodium-selective PVC film of $7.5 \pm 2.5 \mathrm{~nm}$ thickness onto an AFM-tip made of silicon (Ultralevers, Park Scientific Instruments) or silicon nitride (Nanoprobes, Digital Instruments, Santa Barbara, USA) (Fig. 1). In order to avoid coating the whole cantilever, the reflective gold surface was modified with an alkanethiole with hydrophilic terminal groups exposed to the aqueous environment $\left(\mathrm{HS}\left(\mathrm{CH}_{2}\right)_{15} \mathrm{COOH}\right)$ [24]. The sur- face stress induced by this procedure to the cantilever was discussed by Berger et al. in 1997 [25]. The AFM-tip was operated on an artificial substrate (ion-exchanger polymer) releasing potassium or sodium ions specifically to an electrolyte solution. The electrolyte in solution had to enable the ion-exchange at the substrate level and served to conserve electroneutrality of both phases, the ion-exchanger substrate and the polymer-solution boundary of the tip.

Force $v s$. distance curves were acquired with a scan rate of $10 \mathrm{~Hz}$ using a Bioscope AFM-instrument (Digital Instruments, Santa Barbara, CA). An important factor for validating the results was to evaluate the rate limiting process of the ion exchange relative to the scan rate. Diffusion-controlled processes were assumed. Calculations showed that a potassium ion should

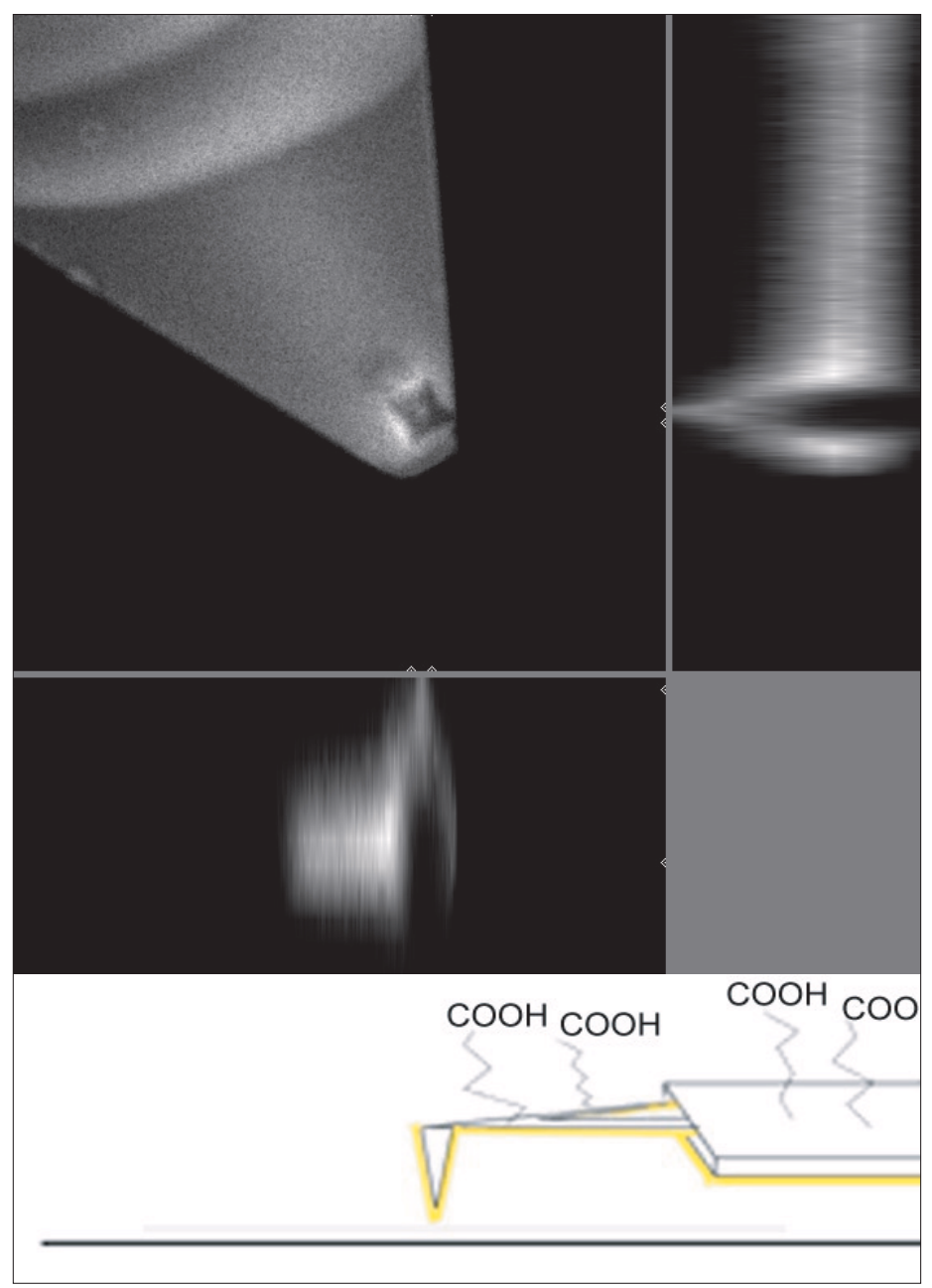

Fig 1. AFM tip coated with a polymer film containing the fluorescent ionophore ETH 7075 (4',5'-dibromofluorescin octadecyl ester, Fluka Chemie AG, Buchs, $\lambda_{\text {em }}=562 \mathrm{~nm}$ ). Cantilever from Nanoprobes, Digital instruments, Santa Barbara, USA. The gold surface was coated with hydrophilic alkanethiols to avoid polymer coating and to conserve the reflectivity (graphical sketch according to [24]). The pictures were taken with a Confocal Laser Scanning Microscope (CLSM) from Leica (Leica, Germany) and elaborated with Imaris Software, Switzerland. The side views show complete coating of the tip-apex but no fluorescence at the reflecting back-side of the cantilever. 
cross $1 \mathrm{~nm}$ distance in aqueous solution within $0.25 \mathrm{~ns}$, which is by orders of magnitude faster than the scanning frequency. Within the polymer substrate, the diffusion rate of $\mathrm{K}^{+}$was estimated to be $1.7 \times 10^{-6} \mathrm{~s}$ per $\mathrm{nm}$. In comparison, the cantilever moves along the surface with a speed of 20 micrometers per second and is displaced by $1 \mathrm{~nm}$ within $5 \times 10^{-5} \mathrm{~s}$, which is more than 30 times slower than the diffusion of alkaline ions within the polymer.

The nominal spring constant of the cantilever of $0.06 \mathrm{~N} \mathrm{~m}^{-1}$ increased by a factor of 15 for the coated and chemically modified cantilever; it was calibrated according to Gibson and Watson in aqueous solution [26]. Despite this increased spring constant, adhesion forces could be accurately measured as a sum parameter involving the interactive area of the AFM-tip [23]. The force-distance curves showed the typical deflection motion and indentation of an AFM-tip on a soft substrate. However, tips coated with an ion-selective film showed increasing stiffness with increasing concentration of the ionophore and the electrolyte solution. Typical elastic behaviour was noticed upon retraction of the tip. The cantilever deflection in the retraction phase was transformed into the quantity of adhesion forces. The net adhesion force was calculated from the difference between the cantilever's adhesion force on a specific substrate releasing potassium or sodium ions and a blank PVC substrate. To distinguish Van der Waals from electrostatic forces was not in the scope of the project. Nevertheless, the interrelationship between adhesion force and concentration allowed the conclusion to be drawn that specific electrostatic interactions were involved. The boundary of the modified AFM-tip was polarized by the selective extraction of potassium or sodium ions, and interacted with the boundary potential of the substrate which released the same specific ion in exchange with the electrolyte solution. Electroneutrality within the substrate phase was guaranteed by adding a lipophilic counter-anion which was not exchanged with chloride of the electrolyte. This concept served as a model to simulate the ion exchange over ion channels. The influence of the ionic strength of the electrolyte and the interrelationship between adhesion force and selectivity of the ion-selective ligand incorporated into the polymer film, which served to coat the modified AFM-tip, provided strong evidence that the assumptions were correct [14]. The ion-exchange and polarization process was fully reversible and was therefore compared to a sodium-potassium pump exchanging both ions reversibly over the two boundaries even though the cantilever motion is the driving force. This process controls the indentation, adhesion and retraction force of the chemically modified AFM-tip.

\section{Application of the Model to $\mathrm{BK}_{\mathrm{Ca}}$ Channels of Living MDCK-F1 Cells and to Voltage-gated Potassium Channels of Adult Rat Cardiomyocytes (ARC)}

Chemically modified AFM-tips are most attractive to add specificity to the excellent geometrical resolution and the high sensitivity to molecular interactions of the tip. If these outstanding features are combined, the chemical identification of molecules imaged by force microscopy will become feasible and chemical analysis on the molecular level will become reality.

Different approaches to trace organic compounds on the scale of single molecules and to quantify interaction forces were reported. Examples are: (1) to investigate rupture forces of individual $\beta$-cyclodextrin-ferrocene host-guest complexes in aqueous medium [27]; (2) individual biomolecules were localized on sensor surfaces by Kossek et al. [28] based on immunochemical interactions. Recently, Arntz et al. described the label-free detection of creatin kinase and myoglobin by a cantilever array [29] and Lang et al. postulated an array of cantilevers where each cantilever is coated with a different polymer to discriminate various solvent vapors [30]. In 2003, E. Meyer et al. postulated the 'Laboratory on a Tip' [31] which demonstrates the potential of Force Microscopy as an analytical tool.

In addition to these challenging approaches, investigations of ion channels by sensing devices are of major interest and are able to contribute to 'the lab on a tip'. Classical chemical sensors are able to detect ion activities reversibly and provide access to monitoring the ion efflux continuously (for a review see [32]). If ion-channels are traced, the reference method is the PatchClamp technique which was awarded the Nobel Prize in 1992 [33]. Although, in comparison to the modified AFM-tip, the Patch-Clamp electrodes are neither operated selectively nor destruction-free. Both characteristics are outstanding features of the chemically modified nanoprobes discussed here. In a next step we decided to advance to show a realistic application of the technique.

Ion channels naturally incorporated into cell membranes play a key role in communication between cells of all living systems [34]. They are involved in signal transduction over cell membranes and in balancing the physiological gradients of ions and electrolytes in different cell compartments. On the one hand, $\mathrm{Na}^{+}-\mathrm{K}^{+}$-ATPase controls the sodium-potassium gradient and is responsible for balancing the osmotic pressure, water retention and, therefore, the cell volume. On the other hand, calcium-activated potassium channels such as Maxi $\mathrm{K}$ or $\mathrm{BK}_{\mathrm{Ca}}$ channels are found in most ex- citable cell types [35]. In neurons, these calcium-activated potassium channels produce hyperpolarization phases of several seconds. Additionally, Maxi $\mathrm{K}$ channels are known for their large pore-diameter of about $50 \mathrm{~nm}$. Potassium efflux from Maxi $\mathrm{K}$ channels is controlled by calcium ATPase whereas a voltage pulse of $50 \mathrm{mV}$ and calcium concentrations in the medium of 100 $\mathrm{mM}$ stimulate $\mathrm{K}^{+}$-efflux. Inhibitors of this ATPase such as thapsigargin as well as a number of known toxins are able to block the efflux of potassium ions from these 'ligand-operated ion channels' [36]. In addition, a number of drugs, which are relevant to treat diseases such as hypertension by controlling the activity of ion channels, are on the market and are being developed further in the pharmaceutical industry. For this kind of research, the localization of specific ion channels and the monitoring of the activity of ion channels under physiological conditions is an important prerequisite.

AFM as a non-destructive technique was considered to be especially attractive for biological research of living organisms. The ultimate goal of the project was to support drug development and to show the feasibility of localizing potassium channels on living tissues and cell membranes with chemically modified AFM-cantilevers [37]. In addition, with AFM the mapping of the topography may be combined with the mapping of ion channel activity. Most suitable for such investigations are adhesive flatcells which resist to the pressure of the cantilever and have a nuclear region of maximum $10^{-5} \mathrm{~m}$. Cells which fulfill these requirements are differentiated Madin-Darby canine kidney (MDCK-F) cells which are known to express Maxi $\mathrm{K}$ or $\mathrm{BK}_{\mathrm{Ca}}$ channels.

AFM experiments and results of force volume files elaborated as adhesion force maps were compared to immunofluorescence images, to Patch-Clamp electrophysiology experiments and to scans of the topography [14][37]. Ion-channels of living cells were investigated at the Institute of Anatomy at the University of Zürich (Prof. Dr. med. P. Grosscurth) which provided all the necessary facilities and know-how. The Patch-Clamp experiments were operated by Dr. I. Foster, Institute of Physiology, University of Zurich. The results of the immunofluorescent labelling of $\mathrm{BK}_{\mathrm{Ca}}$ channels confirmed the presence of these channels in MDCK-F cells. Patch Clamp experiments combined with experiments applying thaspigargin as a calcium ATPase blocker confirmed the presence and the activity of $\mathrm{BK}_{\mathrm{Ca}}$ channels. Using $\mathrm{K}^{+}$-selective AFM-tips the efflux of potassium ions from these cells could be assessed in vitro with chemically modified AFM-tips. Surprisingly, the resolution of the coated AFM-tip was not the limiting factor for localizing the ion 
channels but rather the pixel size of the imaging technique, which was reduced to $<50$ $\mathrm{nm}$ by collecting an array of $64 \times 64$ force $v s$. distance curves over an area of $3 \times 3 \mu \mathrm{m}^{2}$. All experiments were repeated up to 50 times. Combining the high resolution of the AFM-technique and the high potassium-selectivity of the coating film in physiological solutions, the nanoprobes allowed the observation of ion currents, which were reversibly inhibited by a $2.7 \times 10^{-9} \mathrm{M}$ solution of the Ca-ATPase inhibitor thapsigargin. The cells were imaged in force-volume mode with contact force of $\left\langle 4.2 \times 10^{-9} \mathrm{~N}\right.$ to reduce the mechanical stress for the cell [23]. MDCK-F1 cells are relatively robust and could be investigated for approx. $1 \mathrm{~h}$ before detaching from their substrate. By the way, it was possible to show the migration of MDCK-F1 cells along the surface and to investigate the speed of their movement relative to the scanning speed of the cantilever [14]. Investigations of cells with AFM probes have become more commonplace. However the indentation of the tip into the cell caused problems for the survival of cells. In the case of the coated probes, the active diameter of the tip apex is in the range of $25 \pm 5 \times 10^{-9} \mathrm{~m}$, the thickness of the polymer film of $7.5 \pm 2.5 \times 10^{-9} \mathrm{~m}$ included. Considering the tip apex as a ball-point, the total contact area may be in the range of $490 \pm 216$ $\mathrm{nm}^{2}$. Therefore the mechanical stress and the adhesion force are distributed on a larger area and, as a consequence, they are less destructive.

The final highlight of the Ph.D. thesis of Prisca Zammaretti [14] was to investigate adult rat cardiomyocytes (ARC) by chemically modified cantilevers and to monitor the $\mathrm{K}^{+}$-efflux. Isolation of adult rat cardiomyocytes and cultivation is a complex procedure. In culture, the cells require the addition of serum in order to survive for more than five days. However the structure of muscle cells is strictly organized and the localization of voltage gated ion channels is known to be distributed on the $\mathrm{z}$-lines of the lamellopodium. By staining the $\mathrm{z}$-line epitop of ARC cells, the distribution of z-lines can be visualized using CLSM (confocal laser scanning microscopy) (Fig. 2). The distance between $\mathrm{z}$-lines was shown to be $2.3 \times 10^{-6} \mathrm{~m}$ which means that also ion channels must be found in the same distance of each other. The results acquired with the modified cantilever and transformed into an elasticity map (in $10^{-9} \mathrm{~N}$ ) was compared to the elasticity plot (in $10^{-9} \mathrm{~N}$ ) and the topography (in $10^{-9} \mathrm{~m}$ ) (Fig. 3). Surprisingly, the distance between lines of low elasticity to the cantilever (white pixels in Fig. 3b) coincided with the distance between the $\mathrm{z}$ lines and the distance of high adhesion to the chemically modified AFM-tip. Using a potassium-selective AFM-tip under the same conditions as mentioned above, a dis- tance of $1.8 \pm 0.5 \times 10^{-6} \mathrm{~m}$ was measured (average over 10 different ARC cells imaged at least twice). With high probability, the potassium-selective AFM-tip gave access to localization of the voltage-gated potassium-efflux from opened ion channels on rat cardiomyocytes.

\section{What is Next in the Development of Chemically Selective Nanoprobes at CCS?}

A crucial step when modifying surfaces chemically are the surface properties which allow adsorption of chemical compound or covalent linkage with more or less effort. The most frequently asked questions in the project above was 'why don't you immobilize the ionophore on the surface of the AFM-tip?'. One answer to this question was that the chemical environment of an ionophore plays a crucial role for the preformation of the complexing site and the effective complexation of an ion. More than three decades were spent to find satisfying conditions for a number of ionophores used for medical and biological investigations by incorporating the active compounds into a polymer film.

The adsorption of a polymer film to the silicon and silicon nitride nanostructure of the active surface of the AFM-tip worked surprisingly well and was stable for a few days of operation. Most probably this satisfying performance was correlated to the relatively small coated area and the shape of the cantilever tip. In our experience, the adhesion of chemical coatings relying on physico-chemical adsorption becomes more crucial the bigger the coated area is, in addition to other surface properties such as wettability, roughness etc. These experiences were primarily made over a period of more than ten years when we had been working with optical sensors. Traditionally, silanization was used to control the wettability and contact angle of glass and quartz surfaces. Due to the extremely high sensitivity, this step was found to be much more crucial for planar integrated optical sensors.

However, when investigating chemically modified integrated optical waveguides to trace enzyme activities (active proteomics on chip) and to trace the activity of ions and neutral compounds (classical optical sensors (optodes) on chip), we learnt that a change in strategy is necessary [38]. Currently a number of different immobilization strategies for metal oxide surfaces are under investigation.

\section{Acknowledgements}

To close this summary on bioanalytical and nanotechnological activities at CCS, we acknowledge the financial support by the Swiss National Science Foundation (NFP 36). My thanks go to the Institute of Anatomy at the Uni-
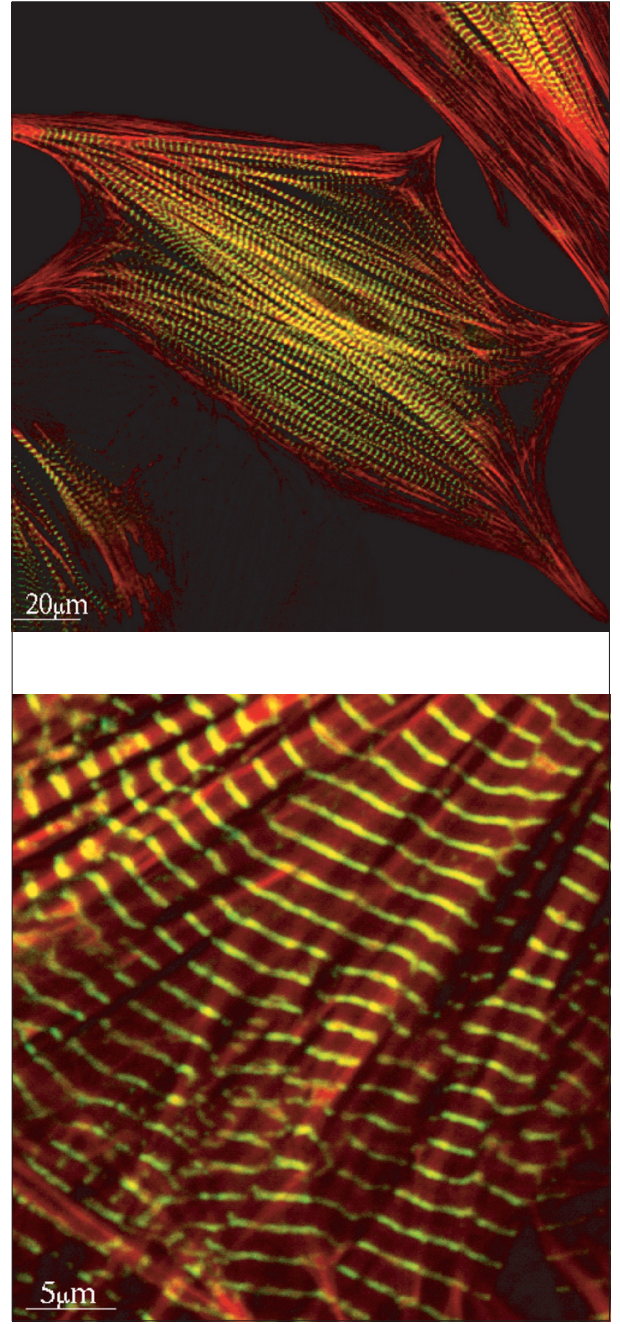

Fig. 2. Formaldehyde fixed ventricular cardiac muscle cells of adult female rats (vARC). $\alpha$-actinin filaments are shown in red, myosin in green due to staining of the $\mathrm{z}$-lines. Maxi $\mathrm{K}$ channels are generally located on z-lines, data not shown. CLSM image courtesy of M. Hefti, Institute of Pharmacology, University of Zurich)

versity of Zurich (Prof. Dr. med. P. Grosscurth and Dr. U. Ziegler) and to other institutes and scientists mentioned in the text who contributed to the success of the project. ARC cells were kindly provided by Prof. M. Schaub and Dr. M. Hefti, Institute of Pharmacology, University of Zurich. Prisca Schär-Zammaretti was an extremely motivated Ph.D. student and did all the experiments and practical work.

Received: August 30, 2004

[1] U.E. Spichiger-Keller, Chimia 2003, 57, 317-320.

[2] U.E. Spichiger-Keller, Chimia 1997, 51, 790-793.

[3] U.E. Spichiger, C. Demuth, A. Fakler, L. Jenny, M. Linnhoff, C. Lohse, G.J. Mohr, A. Moradian, J.P. Müller, S. Nagel, T. Nezel, T. Roth, M. Rothmaier, P. Zammaretti, W. Zhang, G. Khlyak, Chimia 1999, 53, 91-97.

[4] T. Nezel, U.E. Spichiger-Keller, C. Ludin, A. Hensel, I.a.o.S.-P. Winners, Chimia 2001, 55, 725-731. 

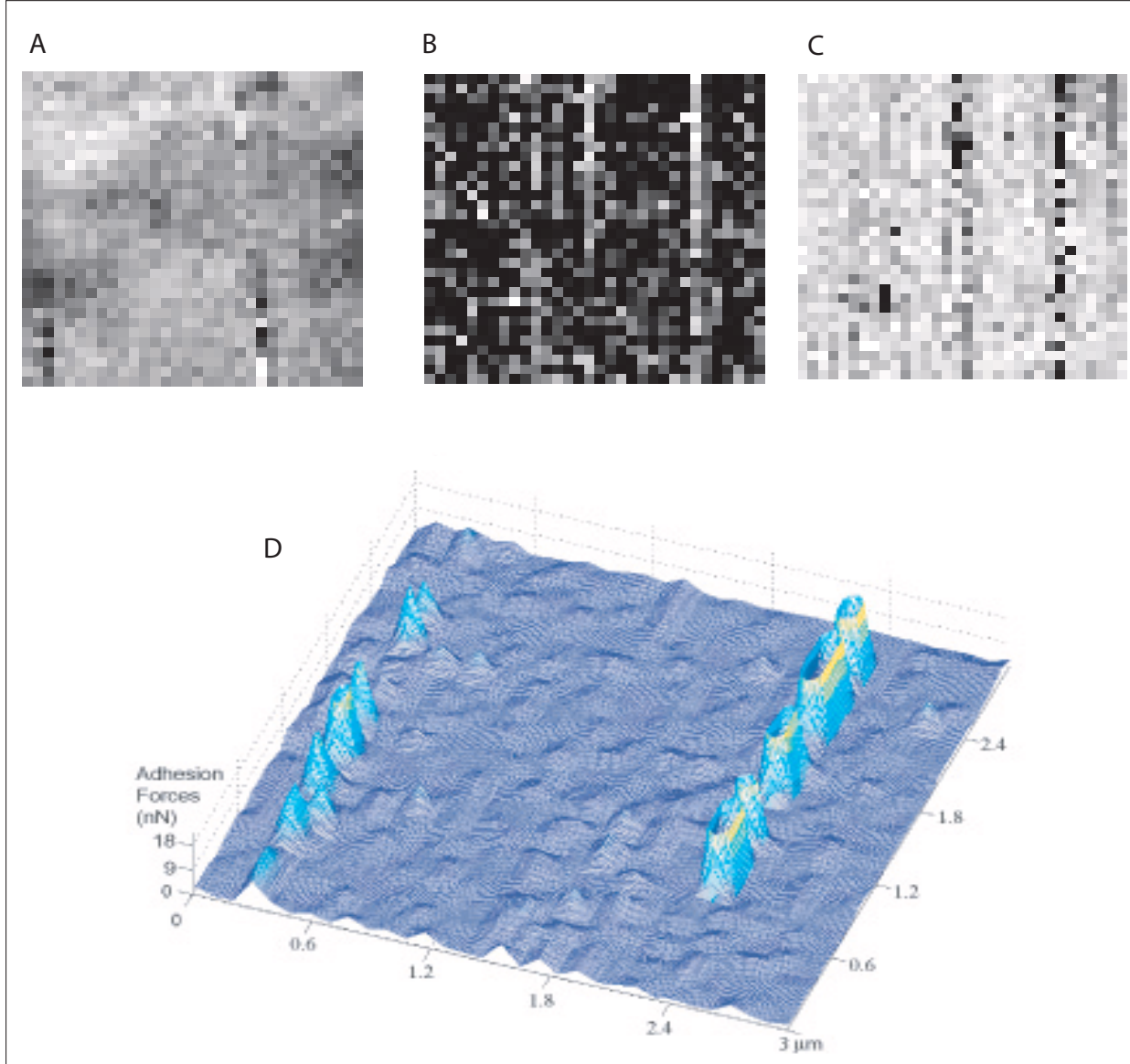

Fig. 3. AFM images $\left(6 \times 6 \mu \mathrm{m}^{2}\right)$ of a part of the lamellopodium of a living ARC cell. A. topography, B. elasticity plot, C. adhesion map monitored with a potassium-selective AFM tip (white pixel, $1.2 \mathrm{nN}$, low adhesion; black pixel $60 \mathrm{nN}$, high adhesion); D. graphical transformation of the adhesion forces referred to image $C$. The opened potassium channels are represented as peaks and are located in lines ( $\mathrm{x}, \mathrm{y}$-axis, scanned area; $\mathrm{z}$-axis, adhesion force in $\mathrm{nN})$. All images were collected with force volume imaging method using a Bioscope (Digital Instruments, USA).

[5] U. Schaller, E. Bakker, U.E. Spichiger, E. Pretsch, Talanta 1994, 41, 1001-1005.

[6] X. Zhang, A. Fakler, U.E. Spichiger, Electroanalysis 1998, 17, 1174-1181.

[7] U.E. Spichiger, A. Fakler, Electrochimica Acta 1997, 42, 3137-3145.

[8] X. Zhang, J. Wang, B. Ogorevc, U.E. Spichiger, Electroanalysis 1999, 11, 945-949.

[9] X. Zhang, A. Fakler, U.E. Spichiger, Anal. Chim. Acta 2001, 445, 57-65.

[10] T. Nezel, U.E. Spichiger-Keller, C. Ludin, A. Hensel, Chimia 2001, 55, 725-731.

[11] U. Spichiger-Keller, J. Hayoz, A. Tschupp, E. Schmid, M. Loher, KTI Project Nr. 5562.2 / SUS, 2001.

[12] W.E. Morf, K. Seiler, B. Lehmann, C. Behringer, S.S.S. Tan, K. Hartman, P.R. Sorensen, W. Simon, in 'Ion-Selective Electrodes, 5', Ed. E. Pungor, Pergamon Press, Oxford, New York, 1988, Vol. 5.

[13] F. Lantner, R.A. Steiner, D. Ammann, W. Simon, Anal. Chim. Acta 1982, 135, 51.

[14] P.S. Zammaretti, 'Chemically Selective Nanoprobes', Swiss Federal Institute of
13805, 2001.

[15] H.G. Hansma, S.A.C. Gould, P.K. Hansma, H.E. Gaub, M.L. Longo, J.A.N. Zasadzinski, Langmuir 1991, 7, 1051-1054.

[16] H.J. Guntherodt, J. Therm. Anal. 1991, 37, 1631-1632.

[17] H.G. Hansma, J. Vesenka, C. Siegerist, G. Kelderman, H. Morrett, R.L. Sinsheimer, V. Elings, C. Bustamante, P.K. Hansma, Science 1992, 256, 1180-1184.

[18] U. Dammer, O. Popescu, P. Wagner, D. Anselmetti, H.J. Guntherodt, G.N. Misevic, Science 1995, 267, 1173-1175.

[19] U. Dammer, M. Hegner, D. Anselmetti, P. Wagner, M. Dreier, W. Huber, H.J. Guntherodt, Biophys. J. 1996, 70, 2437-2441.

[20] K. Feldmann, T. Tervoort, P. Smith, N.D. Spencer, Langmuir 1998, 14, 372-378.

[21] N.V. Lavrik, M.J. Sepaniak, P.G. Datskos, Rev. Sci. Instrum. 2004, 75, 2229-2253.

[22] P.T. Frederix, B.W. Hoogenboom, D. Fotiadis, D.J. Muller, A. Engel, MRS Bull. 2004, 29, 449-455.
[23] P. Zammaretti, A. Fakler, F. Zaugg, U.E. Spichiger-Keller, Anal. Chem. 2000, 72, 3689-3695.

[24] F.G. Zaugg, Swiss Federal Institute of Technology-Ph.D. thesis Nr. ETH Nr.13 182, 1999.

[25] R. Berger, E. Delamarche, H.P. Lang, C. Gerber, J.K. Gimzewski, E. Meyer, H.-J. Güntherodt, Science 1997, 276, 2021.

[26] C.T. Gibson, G.S. Watson, S. Myrha, Nanotechnology 1996, 7, 259-262.

[27] S. Zapotoczny, T. Auletta, M.R. de Jong, H. Schoenherr, J. Huskens, F.C.J.M. van Veggel, D.N. Reinhoudt, G.J. Vancso, Langmuir 2002, 18, 6988-6994.

[28] S. Kossek, C. Padeste, L.X. Tiefenauer, H. Siegenthaler, Biosensors \& Bioelectronics 1998, 13, 31-43.

[29] Y. Arntz, J.D. Seelig, H.P. Lang, J. Zhang, P. Hunziker, J.P. Ramseyer, E. Meyer, M. Hegner, C. Gerber, Nanotechnology 2003, 14, 86-90.

[30] H.P. Lang, M. Hegner, C. Gerber, Chimia 2002, 56, 515-519.

[31] E. Meyer, H.J. Hug, R. Bennewitz, 'Scanning Probe Micsroscopy: The lab on a tip', Springer-Verlag, Berlin, 2003.

[32] L. Luo, X. Yang, E. Wang, Analytical Letters 1999, 32, 1271-1286.

[33] B. Sakmann, EMBO J 1992, 6, 2002-2016.

[34] B. Alberts, 'Molecular biology of the cell', 3rd Edition, Garland Publishing, 1994.

[35] V. Cena, B. Soria, 'Ion channel pharmacology', Oxford University Press, 1998.

[36] E. Mutschler, 'Arzneimittelwirkungen', Wissenschaftliche Verlagsgesellschaft mbH, Stuttgart, 1996.

[37] P. Schär-Zammaretti, U. Ziegler, I. Forster, P. Groscurth, U.E. Spichiger-Keller, Anal. Chem. 2002, 74, 4269-4274.

[38] G. Zhylyak, V. Ramoz-Perez, M. Linnhoff, T. Hug, D. Citterio, U.E. Spichiger-Keller, Optics and Lasers in Engineering 2004, in press. 